\title{
Focused ultrasound stimulation on human language-related acupoints modulates brain activity in cortical language processing regions
}

\author{
Jie $\mathrm{Liu}^{1,2 \dagger}$, Shijun Qiu${ }^{3 \dagger}$, Xin Chen ${ }^{4 \dagger}$, Yulong Zhou ${ }^{1,2}$, Jinjian $\mathrm{Wu}^{5}$, Tong Sun ${ }^{4}$, Di Yuan ${ }^{1,2}$, Haoyue Tian ${ }^{1,2}$, Veronica P. Y. Kwok ${ }^{1,2}$, \\ Joey Tang ${ }^{1,6}$, Li Hai $\operatorname{Tan}^{1,2 *}$ \\ Focused ultrasound provides a more optimised solution than other traditional non-invasive neuromodulation techniques given its superior \\ spatial specificity and penetration depth. However, due to attenuation and dispersion effects, it has not been widely used for human \\ neuromodulation. This study presents a pioneering attempt to activate language-associated brain circuits through the use of focused ultrasound \\ stimulation (FUS) on acupoints in human limbs, thus bypassing the distorting effects. When FUS was delivered to Tongli in the arm and \\ Xuanzhong in the leg, significant increase in FMRI BOLD signal was recorded in language regions including the bilateral insulae and the left \\ inferior parietal lobule. However, when FUS was delivered to the sham acupoint, Waiguan, no significant activation in cortical language regions \\ was evident. We conclude that FUS delivered upon language-related acupoints can be used to modulate human cortical language function, \\ which sheds light on the neurophysiological mechanisms of human language and treatment options for language disorders.
}

Keywords: focused ultrasound, non-invasive neuromodulation, language, brain

\section{INTRODUCTION}

Non-invasive neuromodulation techniques, such as transcranial electrical stimulation (tDCS or tACS) and transcranial magnetic stimulation (TMS), provide opportunities for modulating regional brain activity through either increasing or decreasing of human brain excitability, rendering a wide range of therapeutic applications potentially helpful to the treatment of neurological and neuropsychiatric disorders, as well as the promotion of brain functions in healthy individuals (1). However, these techniques have limited spatial specificity and penetration depth $(2,3)$. In contrast, focused ultrasound (FUS) transmits pressure waves that converge on a selected focal spot with millimetre precision $(4,5)$. FUS has been reported to significantly modulate brain activation in animal models, including intact mice $(6,7)$, rabbits $(8,9)$, sheep $(10)$ and healthy primates (11), without causing tissue damage. However, there are very few available studies in healthy humans (12-15) due to the significant reduction of stimulation effect caused by attenuation, scattering and absorption of ultrasound by the human skull (16).

To overcome the attenuation and dispersion effects of the human skull caused by the impedance mismatch between the skull and underlying layers, we propose to make use of acupuncture points, or acupoints, in other human body parts. Acupuncture, which typically involves needle insertion through the skin into a depth of 15-25 $\mathrm{mm}$ at the acupoints, has been a key component of traditional Chinese medicine, practised for over 3000 years (17). Prior studies have demonstrated that the clinical benefits of acupuncture are mediated by the central nervous system (18-20). Research using blood oxygenation level-dependent functional magnetic resonance imaging (BOLD fMRI) has confirmed that needling acupoints indeed brings about adjustments in cortical and subcortical nerve activity $(21,22)$. By analogy, FUS can be applied to acupoints to induce changes in the central nervous system and human brain activity, in a similar way brought about by acupuncture.

\footnotetext{
${ }^{1}$ Center for Language and Brain, Shenzhen Institute of Neuroscience, Shenzhen, China. ${ }^{2}$ Guangdong Platform of Translational Research for Cerebrovascular Diseases, Shenzhen, China. ${ }^{3}$ Department of Radiology, First Affiliated Hospital of Guangzhou University of Chinese Medicine, Guangzhou, China. ${ }^{4}$ School of Biomedical Engineering, Shenzhen University Health Science Center, Shenzhen, China. ${ }^{5}$ The First School of Clinical Medicine, Guangzhou University of Chinese Medicine, Guangzhou, Guangdong, China. ${ }^{6}$ Myndful Learning Association, Hong Kong SAR, China.

tThese authors contributed equally to this work.

*Corresponding author. Email:tanlh@sions.cn (L.H.T.)
}

Our motivation to demonstrate FUS as a novel neuromodulation method was fuelled by its advantages over acupuncture. Firstly, FUS is non-invasive as opposed to the invasive nature of needling in acupuncture. In practice, the delivery of FUS to acupoints does not require the manual operation of a skilled acupuncturist. Moreover, the signal intensity of FUS can be varied and therefore quantitatively specified for different purposes.

The current study was set out to test the possibility of inducing language-related brain activity changes by stimulating the acupoints implicated in language functions using FUS. According to the Chinese National Standards published in 2006, there is a total of 408 acupoints in the human body (23). Existing studies converge on the finding that acupuncture facilitates the release of specific neuropeptides in the central nervous system, eliciting profound physiological effects and even activating self-healing mechanisms (24). Of the 408 acupoints, the following eighteen are related to language functions: Tongli (HT5), Xuanzhong (GB39), Yamen (DU15), Sanyangluo (SJ8), Fengfu (DU16), Fengchi (GB20), Wangu (GB12), Naohu (DU17), Houxi (SI3), Hegu (LI4), Taichong (LR3), Xinmen (DU22), Shenting (DU24), Baihui (DU20), Jinjin (Ex-HN12), Yuye (Ex-HN13), Lianquan (CV23) and Yongquan (KI1). Previous fMRI research demonstrated that needling language-related acupoints, such as Tongli (HT5) in the inner arm and Xuanzhong (GB39) in the leg, activated cortical language networks $(21,22)$. The present study delivered FUS to these acupoints and assessed its effects on haemodynamic fluctuations using functional magnetic resonance imaging (fMRI).

\section{RESULTS}

Brain activations during the language localiser task

Significant brain activations during the language localiser (synonym judgement) task relative to the resting baseline were reported (see Table 1 and Fig. 1). These clusters (which formed the ROIs used in further analyses below) were located at the following sites: the left lingual gyrus (peak MNI coordinates [-36, -87, -15]), left supplementary motor area (peak MNI coordinates [-6, 9, 54]), left opercular inferior frontal gyrus (peak MNI coordinates [-39, 6, 27]), left insula (peak MNI coordinates [-30, 27, 6]), right insula (peak MNI coordinates [39, 18, 3]), right thalamus (peak MNI coordinates $[15,-18,9]$ ), right precentral gyrus (peak MNI coordinates $[57,-9,51]$ ), left inferior parietal lobule (peak MNI 
coordinates $[-51,-39,57]$, peak MNI coordinates [-33, -63, 57]) and right postcentral gyrus (peak MNI coordinates [33, -45, 66]). This pattern of activation is consistent with previous findings using similar paradigms $(25,26)$.

Table 1. Local maxima of brain activation during the language localiser task (whole brain FDR correction at $p<.01$, cluster level FWE correction at $p<.05$ ).

\begin{tabular}{|c|c|c|c|c|c|c|}
\hline \multirow[t]{2}{*}{ Region } & \multirow{2}{*}{$\begin{array}{l}\text { Brodmann } \\
\text { Area } \\
\text { (BA) }\end{array}$} & \multicolumn{3}{|c|}{ MNI Coordinates } & \multirow{2}{*}{$\begin{array}{l}\text { No. of } \\
\text { Voxels }\end{array}$} & \multirow[t]{2}{*}{ T-value } \\
\hline & & $\mathrm{x}$ & $\mathrm{y}$ & $\mathrm{z}$ & & \\
\hline \multirow[t]{3}{*}{ Left lingual gyrus } & 19 & -36 & -87 & -15 & 2305 & 11.36 \\
\hline & 37 & -27 & -48 & -30 & & 10.90 \\
\hline & 37 & -45 & -60 & -15 & & 9.43 \\
\hline \multirow{3}{*}{$\begin{array}{l}\text { Left supplementary } \\
\text { motor area }\end{array}$} & 6 & -6 & 9 & 54 & 1039 & 9.79 \\
\hline & 32 & 9 & 27 & 36 & & 7.84 \\
\hline & 6 & 12 & 6 & 66 & & 7.29 \\
\hline \multirow{2}{*}{$\begin{array}{l}\text { Left opercular } \\
\text { inferior frontal gyrus }\end{array}$} & 48 & -39 & 6 & 27 & 1351 & 9.08 \\
\hline & 44 & -45 & 6 & 33 & & 8.59 \\
\hline Left insula & 47 & -30 & 27 & 6 & & 8.37 \\
\hline \multirow[t]{3}{*}{ Right insula } & 48 & 39 & 18 & 3 & 253 & 8.08 \\
\hline & 47 & 30 & 27 & 0 & & 7.96 \\
\hline & 48 & 24 & 12 & 9 & & 3.94 \\
\hline \multirow[t]{3}{*}{ Right thalamus } & & 15 & -18 & 9 & 264 & 7.22 \\
\hline & & 18 & 0 & 15 & & 6.16 \\
\hline & & 21 & -12 & 21 & & 5.85 \\
\hline \multirow{3}{*}{$\begin{array}{l}\text { Right precentral } \\
\text { gyrus }\end{array}$} & 4 & 57 & -9 & 51 & 174 & 6.25 \\
\hline & 4 & 54 & -15 & 45 & & 6.04 \\
\hline & 2 & 45 & -30 & 48 & & 5.02 \\
\hline \multirow{2}{*}{$\begin{array}{l}\text { Left inferior parietal } \\
\text { lobule }\end{array}$} & 40 & -51 & -39 & 57 & 92 & 5.95 \\
\hline & 40 & -45 & -36 & 39 & & 5.44 \\
\hline \multirow{3}{*}{$\begin{array}{l}\text { Right postcentral } \\
\text { gyrus }\end{array}$} & 2 & 33 & -45 & 66 & 115 & 5.84 \\
\hline & 40 & 30 & -48 & 45 & & 4.74 \\
\hline & 40 & 27 & -48 & 33 & & 4.62 \\
\hline \multirow{2}{*}{$\begin{array}{l}\text { Left inferior parietal } \\
\text { lobule }\end{array}$} & 7 & -33 & -63 & 57 & 123 & 5.69 \\
\hline & 7 & -30 & -60 & 42 & & 5.54 \\
\hline
\end{tabular}

\section{FUS modulates activation in the cortical language regions}

In a within-participants design, we investigated the impact of FUS stimulation on brain activity in the cortical regions typically associated with language processing. We extracted the mean parametric estimate for each of the ten aforementioned ROIs for both the experimental and the sham stimulation conditions and compared each of these values to corresponding value during the resting baseline. A one sample $t$-test revealed significantly lower activation in the left lingual gyrus in the experimental condition relative to the baseline (parametric estimates $=-0.56 \pm 1.10, t(25)=2.91$, FDR corrected $p<.05)$. Compared to the baseline, FUS stimulation at Tongli and Xuanzhong elicited significantly greater activation in the left insula (parametric estimates $=0.33 \pm 0.13, t(25)=2.59$, FDR corrected $p<.05$ ), right insula (parametric estimates $=0.25 \pm 0.48$, $t(25)=3.00$, FDR corrected $p<.05)$ and left inferior parietal lobule
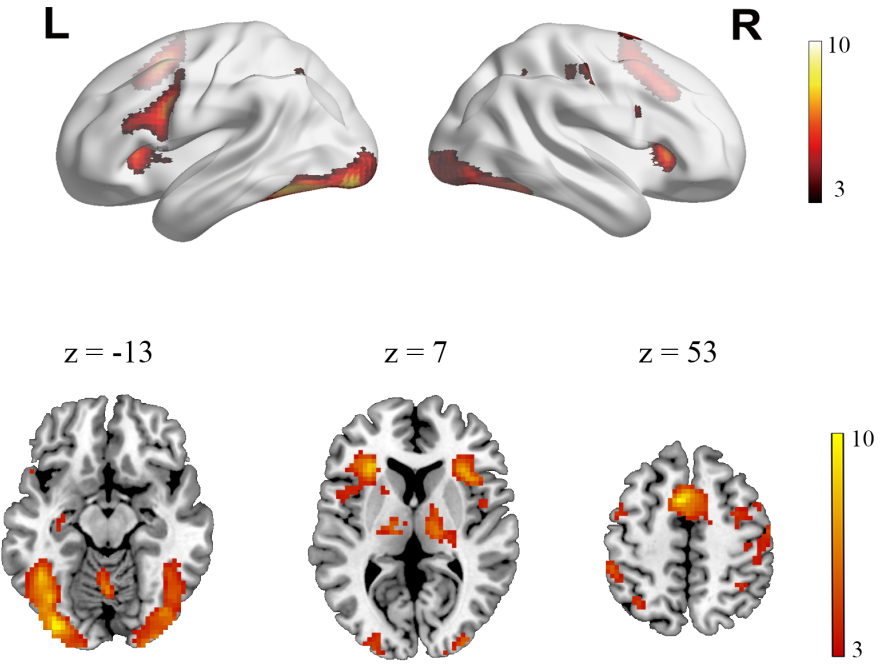

Fig. 1. Brain regions showing significant activation during the language localiser task (whole brain FDR correction at $\boldsymbol{p}<.01$ ). The colour bars refer to T values.

(parametric estimates $=0.35 \pm 0.77, t(25)=2.60$, FDR corrected $p$ $<.05)$. In contrast, FUS stimulation at the sham acupoint, Waiguan, did not evoke any significant difference from the baseline in any of the ROIs ( $p s>.05)$ (see Fig. 2).
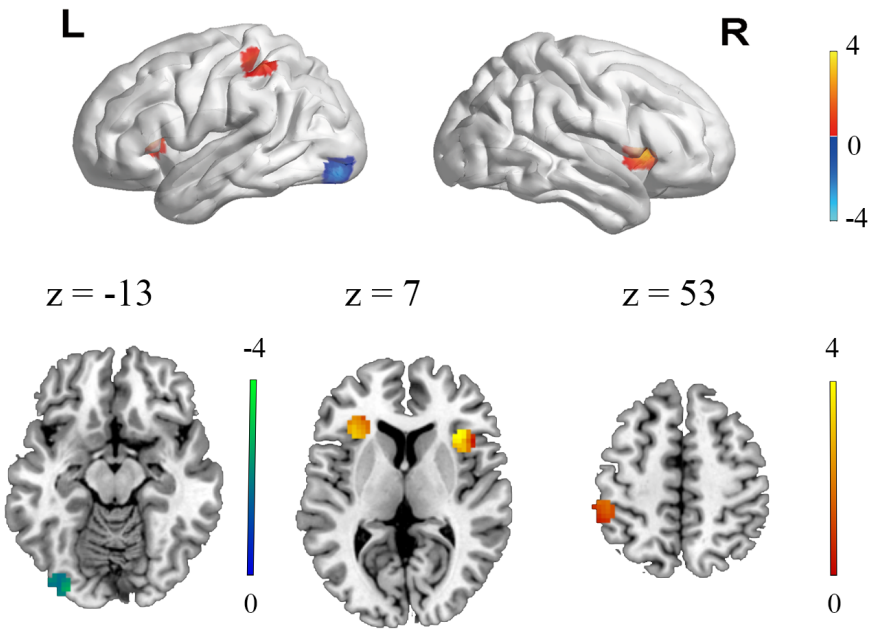

Fig. 2. Cortical regions showing significant activation during FUS stimulation at language-related acupoints (ROI analyses). The colour bars refer to T values.

\section{DISCUSSION}

In this pioneering study, we set out to investigate the effects of stimulating language-implicated acupoints, namely Tongli and Xuanzhong located in human limbs, using FUS on neuromodulation in the language processing circuit. When FUS was applied to these language-implicated acupoints, significant activation was observed in several key brain language regions, including the bilateral insulae and the left inferior parietal lobule, among other brain regions typically associated with language processing. FUS stimulation at the sham acupoint, Waiguan, did not however lead to any significant increase in BOLD signal in any of the typical language-processing regions.

Our neuroimaging data are consistent with the existing literature on human language processing. In particular, the left inferior parietal cortex has been suggested to support the integration of lexical inputs into larger units for semantic processing (27), 
especially in Chinese logograph reading (26). A meta-analysis of 42 fMRI studies with healthy adults showed that the bilateral insulae were significantly activated in both language (expressive and receptive) and speech (production and perception) tasks (28). The importance of the insulae has long been recognised in their reciprocal functional and structural connections with linguistic, motor, limbic and sensory brain areas (29).

Despite greater spatial selectivity and depth penetration compared to other traditional non-invasive techniques, such as transcranial magnetic stimulation (TMS) or transcranial direct-current stimulation (tDCS) $(12,15)$, the application of FUS to human neuromodulation is limited due to the significant reduction of stimulation effect caused by attenuation, scattering and absorption of ultrasound by the human skull (16). The current study demonstrated, for the first time, the possibility of human neuromodulation by applying FUS on language-implicated acupoints located in human limbs, thus bypassing the distorting effects commonly experienced in studies where attempts were made to apply FUS to the human brain through the skull.

Our findings introduce a new perspective of individualised non-invasive neuromodulation as potential treatment for language disorders. In patients with aphasia, cortical activation in the left hemisphere language areas is greatly reduced and later language improvement is typically associated with an increase or "reshift" of cortical activity to perilesional areas (30). Transcranial magnetic and electric stimulations (i.e., TMS and tDCS) have frequently been combined with behavioural intervention in language rehabilitation. Despite the fact that tDCS and TMS are successful in enhancing activity in language regions in both patients with aphasia $(31,32)$ and healthy participants (30), these methods could also lead to harmful consequences, such as seizures $(33,34)$. In contrast, there is no reported harm to human health from using ultrasound (except minimal pain experienced by some individuals) and no reported tissue damage in studies implementing its nonthermal bioeffects on neuromodulation $(8,35)$. Additionally, the use of tDCS or TMS requires MRI guidance to provide individualised and precise localisation of target brain region for stimulation which is often costly and impractical. In comparison, not only does FUS allow greater spatial selectivity and depth penetration, it does not require MRI scanning to guide brain localisation as stimulation can be delivered to acupoints located in human limbs, thus rendering a much more cost-effective and convenient treatment option.

Previous fMRI studies have demonstrated that needling language-implicated acupoints evoked significant activation in cortical language areas. For instance, Liu et al. (2017) observed enhanced activation of multiple language regions, including the left insula, the opercular and triangular parts of the left inferior frontal gyrus and the bilateral precentral gyri upon the needling of Tongli in the right arm (36). Likewise, Li et al. (2011) reported enhanced activation in the right inferior frontal gyrus and the bilateral superior temporal gyri upon electroacupuncture (a form of acupuncture where a small electric current is passed between pairs of acupuncture needles) on language-implicated acupoints Sanyangluo (SJ8; located in the wrist) and Yamen (DU15; located under the first cervical vertebra) respectively (37). Greater increase in activation in the right insula was also observed upon application of electroacupuncture to post-stroke aphasic patients compared to healthy controls (37). The enhanced activation in the bilateral insulae following FUS stimulation at the two language-implicated acupoints Tongli and Xuanzhong demonstrated in our current study is in harmony with the acupuncture literature. We speculate that neuromodulatory effects elicited by FUS stimulation at acupoints share, at least partially, the same underlying neurophysiological mechanisms with those elicited by acupuncture. In addition, the signal intensity of FUS can be varied and may therefore provide a better personalised treatment technique compared to acupuncture, the intensity of which cannot be varied. Future research should focus on optimising FUS parameters or exploring more effective stimulation methods in order to produce long-lasting neuromodulatory effects for language treatment purposes, reflected by improved language-related behavioural performance.

Through the coupling of modern biomedical engineering technology and traditional Chinese medicine principles, our current study has demonstrated a novel method of applying focused ultrasound on language-implicated acupoints for human neuromodulation. Significant increase in BOLD signal was observed in several key brain language regions, including the bilateral insulae and the left inferior parietal lobule when focused ultrasound was delivered to the two acupoints, namely Tongli and Xuanzhong, located in human limbs, thus bypassing the distorting effects through the human skull. Our findings shed light on the neurophysiological mechanisms of human language and treatment options for language disorders.

\section{MATERIALS AND METHODS}

\section{FUS waveform}

The ultrasonic rig used in the present study which included a twochannel, 100-MHz function generator (AFG3102, Tektronics Inc., USA) and a 150-W linear RF amplifier (RF Power Amplifier A150, Electronics \& innovation Inc., New York, NY, USA) connected by a cable to a focused ultrasound (FUS) transducer (Blatek, Inc., State College, PA) placed in the control room adjacent to the MRI scanner room (see Fig. 3). A MATLAB programme was used to control the channel $1 \mathrm{on} / \mathrm{off}$ trigger of the function generator. The parameters of the FUS waveform employed for the current study were as follows: acoustic frequency $(\mathrm{Af})=0.5 \mathrm{MHz}$, pulse duration $(\mathrm{PD})=600 \mu \mathrm{s}$, pulse repetition frequency $(\mathrm{PRF})=1.0 \mathrm{kHz}$, number of pulse $(\mathrm{np})$ $=46000$ and number of cycles per pulse $(\mathrm{c} / \mathrm{p}=300)$. The output of channel 1 was sent via the RF amplifier to the transducer.

The focused ultrasound transducer had a diameter of $30 \mathrm{~mm}$ and a focal length of $30 \mathrm{~mm}$. The ultrasound beam used had a centre frequency of $0.5 \mathrm{MHz}$. The lateral and axial spatial resolutions of the beam were $4 \mathrm{~mm}$ and $1.5 \mathrm{~mm}$ respectively. During the Tongli, Xuanzhong and Waiguan stimulation, the mean voltages were $109.19 \mathrm{mVpp}( \pm 23.31 \mathrm{mVpp}), 152.03 \mathrm{mVpp}( \pm 24.62 \mathrm{mVpp})$ and $102.74 \mathrm{mVpp}( \pm 23.41 \mathrm{mVpp})$ respectively, yielding peak rarefactional pressure values of $1.69 \mathrm{MPa}, 2.35 \mathrm{MPa}$ and 1.59 MPa correspondingly. The spatial-peak pulse-average intensities before transcutaneous transmission for the three acupoints were $57.67 \mathrm{~W} / \mathrm{cm}^{2}, 111.80 \mathrm{~W} / \mathrm{cm}^{2}$ and $51.06 \mathrm{~W} / \mathrm{cm}^{2}$ respectively. The transducer was coated with acoustic coupling gel and affixed on the pre-defined acupoints with medical tape. Due to substantial inter-individual variability in response to ultrasound stimulation, the spatial-peak pulse-average intensity $\left(I_{S P P A}\right)$ used at each acupoint for each participant was determined based on individuals' pain threshold. Each participant's stimulation intensity was personalised and set at $10 \mathrm{mVpp}$ below the intensity at which he/she reported pain at the corresponding acupoint.

\section{Participants}

Twenty-six college students participated in the experiment (12 females and 14 males; mean age $=21.82$ years, $\mathrm{SD}=3.57$ ). All of 


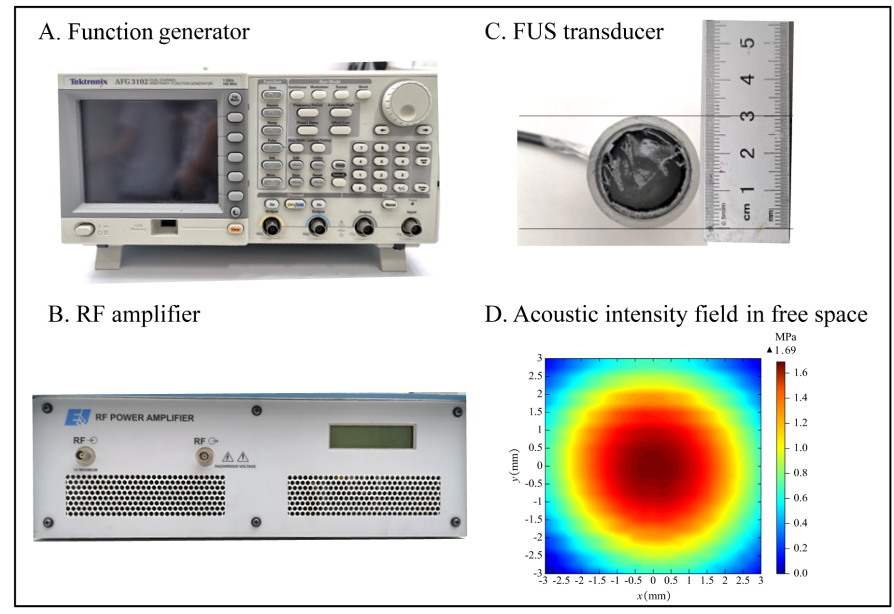

Fig. 3. Ultrasonic rig used in the current experiment (A-C). The function generator (A) was used to configure the ultrasonic signal parameters. The RF amplifier (B) received an input voltage waveform from the function generator to provide output power for the FUS transducer (C) which produced the acoustic pressure profile of the stimulus waveform. (D) Acoustic intensity field in free space.
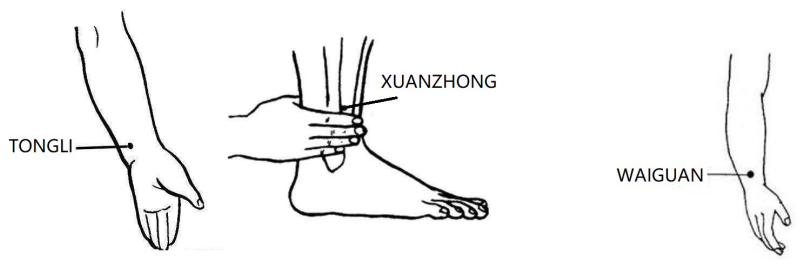

Fig. 4. Anatomical locations of the Tongli (HT5), Xuanzhong (GB39) and Waiguan (TE5) acupoints.

them were strongly right-handed according to a dexterity inventory which required participants to rate their hand preference on nine unimanual tasks (38) using a 5-point Likert-type scale (from $1=$ exclusive left-hand use to $5=$ exclusive right-hand use). Participants who scored more than 40 were defined as strongly right-handed. They had normal or corrected-to-normal vision, with no alcohol or substance abuse or dependence. They were physically healthy and free from psychiatric or neurological disorders. All participants provided written informed consent prior to the experiment. Ethical approval was obtained from the Shenzhen Institute of Neuroscience.

\section{Experimental design and setup}

Three acupoints, Tongli (HT5), Xuanzhong (GB39) and Waiguan (TE5) on the right side of the body were selected for FUS stimulation (Fig. 4). Tongli (HT5) located in the inner arm near the wrist and Xuanzhong (GB39) located in the lower part of the leg are language-implicated acupoints, where acupuncture has demonstrated beneficial roles in the treatment of aphasia $(21,22$, 39), while Waiguan (TE5) - a site typically used by acupuncturists to relieve symptoms of common cold and/or body aches - located in the outer arm near the wrist was not related to language functions and thus served as the sham condition.

The experiment consisted of three runs in the MRI scanner. There were two experimental runs which stimulated Tongli and Xuanzhong, and a sham run which stimulated Waiguan. The order of the experimental and sham conditions was randomised across participants. Each of the experimental runs was organised into four 46-s stimulation blocks, alternated with five 30-s rest blocks. There was a 2-s instruction period at the beginning of each block to allow the participant to get ready (Fig. 5). The sham run consisted of three 46-s stimulation blocks, alternated with four 30s-rest blocks.

After completing the experimental and sham runs, participants were asked to perform a language localiser task (as described below).

\section{The localiser task of language processing}

A typical language task related to the neural basis of semantic processing was adopted for localising purposes in this experiment (26). Twelve pairs of Chinese synonymous characters and 12 pairs of non-synonymous characters were used in this localiser task. The stimuli were shown through an LCD projector system during fMRI scanning. Participants were required to judge whether the two characters in each pair were synonyms or not and respond by pressing a pre-defined button with their left hand. In each trial, a pair of characters was displayed synchronously for $1500 \mathrm{~ms}$, one above and one below a fixation crosshair. After the presentation of character pair, the fixation crosshair remained on for another $1500 \mathrm{~ms}$. Participants were asked to perform the task as quickly and accurately as possible. A block design, consisting of three experimental blocks of synonym judgement alternated with two rest blocks, was adopted. The presentation of synonymous and nonsynonymous characters was randomised within each of the three 24-s experimental blocks. In each of the two rest blocks, a fixation crosshair was displayed for $20 \mathrm{~s}$. A 2-s instruction appeared prior to each block. All participants received a training session prior to scanning to ensure that they understood the instructions of the task.

\section{Functional magnetic resonance imaging data acquisition}

Images were acquired on a Siemens (Munich, Germany) 3T Prisma scanner using a standard 64-channel head coil. After automatic shimming of the magnetic field, three-dimensional (3D) highresolution $\mathrm{T} 1$ anatomical images were acquired for coregistration with the functional images. Functional volumes were acquired using a multiple slice T2*-weighted echo planar imaging (EPI) sequence with the following parameters: repetition time $=2,000$ $\mathrm{ms}$; echo time $=30 \mathrm{~ms}$; flip angle $=90^{\circ}$; matrix dimensions $=64 \times 64 ;$ field of view $=100 \mathrm{~mm}$; slice thickness $=3.5$ $\mathrm{mm}$. Thirty-three slices covered the entire brain. High-resolution three dimensional T1-weighted images were acquired using the magnetisation-prepared rapid gradient-echo sequence (repetition time $=2,530 \mathrm{~ms}$; echo time $=2.98 \mathrm{~ms}$; inversion time $=1,100 \mathrm{~ms}$; flip angle $=7^{\circ}$; field of view $=256 \mathrm{~mm} \times 224 \mathrm{~mm}$; matrix size $=224 \times 256$, interpolated to $448 \times 512,192$ sagittal slices; slice thickness $=1.0 \mathrm{~mm}$, voxel size $=0.5 \times 0.5 \times 1 \mathrm{~mm}$ ).

\section{FMRI data preprocessing}

MRI data were preprocessed and analysed using the SPM12 software (Wellcome Department of Imaging Neurosciences, University College London, UK, http://www.fil.ion.ucl.ac.uk/spm). All volumes were realigned to the first volume and spatially normalised to a common value in order to correct for whole brain differences over time. Images were then smoothed using an isotropic Gaussian kernel of $6 \mathrm{~mm}$ and high-pass filtered at a cut-off of $128 \mathrm{~s}$. The functional images were normalised for each individual with a spatial resolution of $3 \times 3 \times 3 \mathrm{~mm}^{3}$.

\section{Brain activations during the language localiser task}

Statistical analysis was performed by fitting a signal time course for each voxel using the general linear model (GLM). We first calculated the brain activations for the language task relative 
Instruction $2 \mathrm{~s}$

Tongli

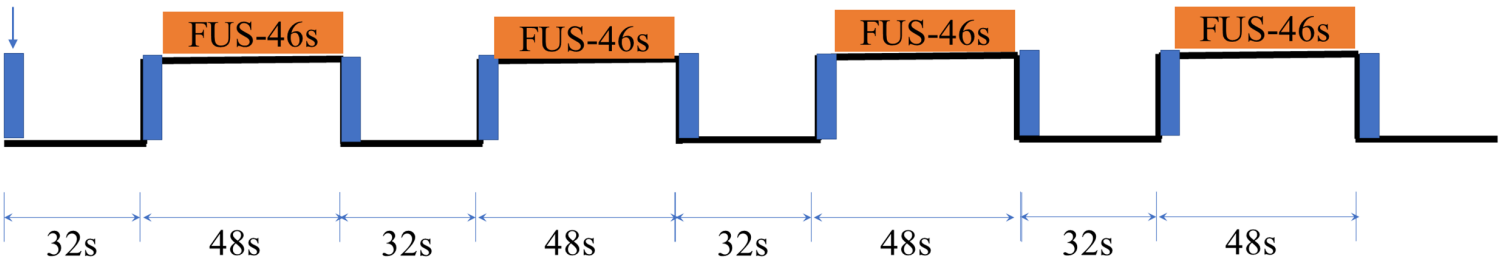

Instruction $2 \mathrm{~s}$

Xuanzhong

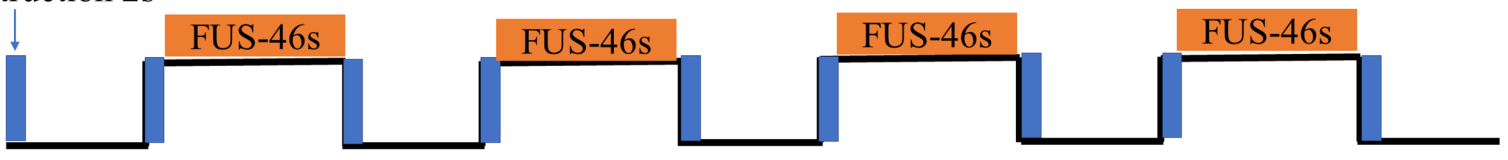

Instruction $2 \mathrm{~s}$

Sham-Waiguan

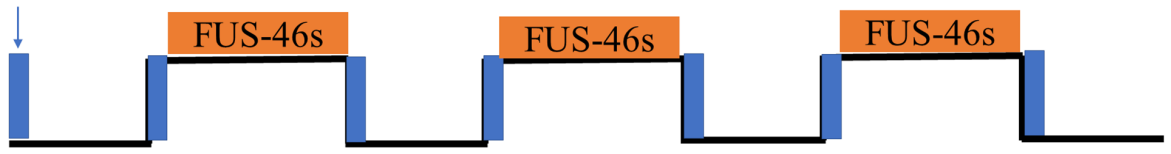

Fig. 5. Block design. The experiment consisted of three runs in the MRI scanner. There were two experimental runs which stimulated Tongli and Xuanzhong, and a sham run which stimulated Waiguan. Each of the experimental runs was organised into four 46-s stimulation blocks, alternated with five 30-s rest blocks, whereas the sham run consisted of three 46-s stimulation blocks, alternated with four 30s-rest blocks. There was a 2-s instruction period at the beginning of each block to allow the participant to get ready.

to rest and obtained parameter-estimated images for individual participants across the whole brain. Then we conducted group analyses with random effects by applying one sample $t$-tests using SPM12 on the brain activation maps of all participants. Significance thresholds were set at $p<.01$ with False Discovery Rate (FDR) correction for whole-brain analyses and at $p<.05$ with Family Wise Error correction for cluster-level analyses respectively. Brain maps were visualised using the BrainNet toolbox for SPM12 (40).

\section{Definition of regions of interest (ROIs)}

Our primary interest was to investigate the effects of FUS stimulation at language-implicated acupoints Tongli and Xuanzhong on brain activity in the associated cortical language regions. The regions of interest (ROIs) were defined as an $8 \mathrm{~mm}^{3}$ sphere around the coordinates of the ten aforementioned significant peaks of activity elicited by the language localiser task (see Table 1). We extracted the mean parametric estimate for each of our ROIs in both experimental and sham stimulation conditions and compared each of them to the corresponding value during the resting baseline. False discovery rate (FDR) correction was used in the ROI analyses.

\section{REFERENCES}

1. E. Dayan, N. Censor, E. R. Buch, M. Sandrini, L. G. Cohen, Noninvasive brain stimulation: From physiology to network dynamics and back. Nat. Neurosci. 16, 838-844 (2013).

2. K. E. Hoy, P. B. Fitzgerald, Brain stimulation in psychiatry and its effects on cognition. Nat.Rev. Neurol. 6, 267-275 (2010).

3. A. Thielscher, A. Opitz, M. Windhoff, Impact of the gyral geometry on the electric field induced by transcranial magnetic stimulation. Neurolmage 54, 234-243 (2011).

4. D. G. Fisher, R. J. Price, Recent advances in the use of focused ultrasound for magnetic resonance image-guided therapeutic nanoparticle delivery to the central nervous system. Front. in Pharmacol. 10, 1348 (2019).

5. F. A. Jolesz, K. Hynynen, N. McDannold, C. Tempany, MR imaging-controlled focused ultrasound ablation: A noninvasive image-guided surgery. Magn. Reson. Imaging Clin. N. Am. 13, 545-560 (2005)

6. P. C. Chu, H. L. Liu, H. Y. Lai, C. Y. Lin, H. C. Tsai, Y. C. Pei, Neuromodulation accompanying focused ultrasound-induced blood-brain barrier opening. Sci. Rep. 5, 15477 (2015).

7. H. A. Kamimura, S. Wang, H. Chen, Q. Wang, C. Aurup, C. Acosta, A. A. O. Carneiro, E. E. Konofagou, Focused ultrasound neuromodulation of cortical and subcortical brain structures using $1.9 \mathrm{MHz}$. Med. Phys. 43, 5730-5735 (2016).

8. K. Hynynen, G. T. Clement, N. McDannold, N. Vykhodtseva, R. King, P. J. White, S. Vitek F. A. Jolesz, 500-element ultrasound phased array system for noninvasive focal surgery of the brain: A preliminary rabbit study with ex vivo human skulls. Magn. Reson. Med. 52, 100-107
(2004)

9. S.-S. Yoo, A. Bystritsky, J.-H. Lee, Y. Zhang, K. Fischer, B.-K. Min, N. J. McDannold, A. PascualLeone, F. A. Jolesz, Focused ultrasound modulates region-specific brain activity. Neurolmage 56, 1267-1275 (2011)

10. W. Lee, S. D. Lee, M. Y. Park, L. Foley, E. Purcell-Estabrook, H. Kim, K. Fischer, L.-S. Maeng S.-S. Yoo, Image-guided focused ultrasound-mediated regional brain stimulation in sheep. Ultrasound Med. Biol. 42, 459-470 (2016).

11. V. Chaplin, M. A. Phipps, C. F. Caskey, A random phased-array for MR-guided transcrania ultrasound neuromodulation in non-human primates. Phys. Med. Biol. 63, 105016 (2018).

12. W. Legon, T. F. Sato, A. Opitz, J. Mueller, A. Barbour, A. Williams, W. J. Tyler, Transcrania focused ultrasound modulates the activity of primary somatosensory cortex in humans. Nat. Neurosci. 17, 322-329 (2014)

13. W. Legon, L. Ai, P. Bansal, J. K. Mueller, Neuromodulation with single-element transcranial focused ultrasound in human thalamus. Hum. Brain Mapp. 39, 1995-2006 (2018).

14. W. Lee, H. Kim, Y. Jung, I. U. Song, Y. A. Chung, S. S. Yoo, Image-guided transcranial focused ultrasound stimulates human primary somatosensory cortex. Sci. Rep. 5, 8743 (2015).

15. W. Lee, Y. A. Chung, Y. Jung, I. U. Song, S. S. Yoo, Simultaneous acoustic stimulation of human primary and secondary somatosensory cortices using transcranial focused ultrasound. $B M C$ Neurosci. 17, 68 (2016).

16. G. Pinton, J.-F. Aubry, E. Bossy, M. Muller, M. Pernot, M. Tanter, Attenuation, scattering, and absorption of ultrasound in the skull bone. Med. Phys. 39, 299-307 (2011).

17. G. Stux, B. Pomeranz, Basics of Acupuncture (Springer, New York, 1998).

18. J. S. Han, G. X. Xie, Z. F. Zhou, R. Folkesson, L. Terenius, Enkephalin and beta-endorphin as mediators of electro-acupuncture analgesia in rabbits: An antiserum microinjection study. Adv. Biochem. Psychopharmacol. 33, 369-377 (1982).

19. G. Litscher, G. Schwarz, A. Sandner-Kiesling, I. Hadolt, E. Eger, Effects of acupuncture on the oxygenation of cerebral tissue. Neurol. Res. 20, S28-S32 (1998).

20. T. Takahashi, Mechanism of acupuncture on neuromodulation in the gut-a review. Neuromodulation 14, 8-12 (2011).

21. L. Bai, W. Qin, J. Tian, P. Liu, L. Li, P. Chen, Y. Liu, Time-varied characteristics of acupuncture effects in fMRI studies. Hum. Brain Mapp. 30, 3445-3460 (2009).

22. L. Bai, J. Tian, C. Zhong, T. Xue, Z. Liu, P. Chen, Y. Liu, Acupuncture modulates temporal neural responses in wide brain networks: Evidence from fMRI study. Mol. Pain 6, 73 (2010).

23. AQSIQ, SAC, Nomenclature and Location of Acupuncture Points (GB/T 12346-2006). (The General Administration of Quality Supervision, Inspection and Quarantine of the People's Republic of China (AQSIQ) and Standardisation Administration of the People's Republic of China (SAC), 2006)

24. J. S. Han, Acupuncture: Neuropeptide release produced by electrical stimulation of different frequencies. Trends Neurosci. 26, 17-22 (2003).

25. W. Hu, H. L. Lee, Q. Zhang, T. Liu, L. B. Geng, M. L. Seghier, C. Shakeshaft, T. Twomey, D. W. Green, Y. M. Yang, C. J. Price, Developmental dyslexia in Chinese and English populations: Dissociating the effect of dyslexia from language differences. Brain 133, 1694-1706 (2010).

26. L. H. Tan, H. L. Liu, C. A. Perfetti, J. A. Spinks, P. T. Fox, J. H. Gao, The neural system underlying Chinese logograph reading. Neurolmage 13, 836-846 (2001).

27. C. K. Thompson, B. Bonakdarpour, S. C. Fix, H. K. Blumenfeld, T. B. Parrish, D. R. Gitelman, M. M. Mesulam, Neural correlates of verb argument structure processing. J. Cogn. Neurosci. 19, 1753-1767 (2007)

28. A. Oh, E. G. Duerden, E. W. Pang, The role of the insula in speech and language processing. Brain Lang. 135, 96-103 (2014). 
29. J. R. Augustine, Circuitry and functional aspects of the insular lobe in primates including humans. Brain Res. Rev. 22, 229-244 (1996)

30. D. Saur, R. Lange, A. Baumgaertner, V. Schraknepper, K. Willmes, M. Rijntjes, C. Weiller, Dynamics of language reorganization after stroke. Brain 129, 1371-1384 (2006).

31. R. Darkow, A. Martin, A. Würtz, A. Flöel, M. Meinzer, Transcranial direct current stimulation effects on neural processing in post-stroke aphasia. Hum. Brain Mapp. 38, 1518-1531 (2017).

32. R. Holland, J. Crinion, Can tDCS enhance treatment of aphasia after stroke? Aphasiology 26, 1169-1191 (2012).

33. J. Classen, O. W. Witte, G. Schlaug, R. J. Seitz, H. Holthausen, R. Benecke, Epileptic seizures triggered directly by focal transcranial magnetic stimulation. Electroen. Clin. Neuro. 94, 19-25 (1995)

34. B. Ekici, Transcranial direct current stimulation-Induced seizure: Analysis of a case. Clin. EEG Neurosci. 46, 169 (2015).

35. Y. Tufail, A. Yoshihiro, S. Pati, M. M. Li, W. J. Tyler, Ultrasonic neuromodulation by brain stimulation with transcranial ultrasound. Nat. Protoc. 6, 1453-1470 (2011).

36. S. Liu, M. Li, W. Tang, G. Wang, Y. Lv, An fMRI study of the effects on normal language areas when acupuncturing the Tongli (HT5) and Xuanzhong (GB39) acupoints. J. Int. Med. Res. 45, 1961-1975 (2017).

37. G. Li, E. S. Yang, An fMRI study of acupuncture-induced brain activation of aphasia stroke patients. Complement. Ther. Med. 19, 49-59 (2011).

38. M. Annett, The binomial distribution of right, mixed and left handedness. Q. J. Exp. Psychol. 19, 327-333 (1967).

39. G. Li, H. L. R. T. Liu, Y. C. Cheung, K. K. Hung, G. G. Wong, E. S. Shen, Yang, An fMRI study comparing brain activation between word generation and electrical stimulation of languageimplicated acupoints. Hum. Brain Mapp. 18, 233-238 (2003).

40. M. Xia, J. Wang, Y. He, BrainNet Viewer: A network visualization tool for human brain connectomics. PIOS ONE 8, e68910 (2013).

Acknowledgments: Funding: This work was supported by grants from Guangdong Key Basic Research Scheme (2018B030332001), Shenzhen Double Chain Grant ([2018]256), Guangdong Pearl River Talents Plan (2016ZT06S220), Shenzhen Peacock Plan (KOTD 2015033016104926) China's National Strategic Basic Research Programme (2012CB720701) and National Natura Science Foundation of China $(81920108019,91649117,81771344)$. Author contributions: J. Liu, Y.L. Zhou, J.J. Wu, T. Sun, D. Yuan, H.Y. Tian collected the data. L.H. Tan designed the experiment J. Liu and V.P.Y. Kwok analysed the data. J. Liu, V.P.Y. Kwok, S.J. Qiu, X. Chen, J. Tang and L.H. Tan drafted the manuscript. Competing interests: All authors declared no competing interests. Data and materials availability: The data and materials of this experiment could be made available upon request to the corresponding author. 